\title{
Portable Dynamic Set-up with Diffusion Type Gas Source for the Calibration of Gas Sensors and Analytic Instruments
}

\author{
A.V.Sokolov ${ }^{1}$, A.L.Laptev ${ }^{2}, \underline{\text { A.A. } \text { Vasiliev }^{1} \text {, A.S. Lagutin }}{ }^{1}$ \\ ${ }^{1}$ NRC "Kurchatov Institute", Kurchatov sq., 1, 123182, Moscow, Russia, \\ Corresponding e-mail address: A-A-Vasiliev@yandex.ru \\ 2"PGS-Analytic", Kirovochepetsk, Kirov region, Russia
}

\begin{abstract}
Portable dynamic set-up is designed for the calibration of gas sensors and gas analytical instruments „in situ“, that is in the place, where these sensors or instruments are installed (laboratory, factory, ecological monitoring system etc.). The set up uses ambient air for the preparation of calibration gas mixture and is equipped with air pump, catalytic filter removing undesirable impurities from ambient air, and diffusion source of target gas. Using this set-up, calibration gas mixture with compounds, which could be liquefied at normal temperature $\left(\mathrm{NH}_{3}, \mathrm{NO}_{2}, \mathrm{Cl}_{2}, \mathrm{HCl}\right.$, formaldehyde, ...), are prepared with precision of $1-4 \%$. The set-up powered by rechargeable battery enables the operation during at least 4 hours.
\end{abstract}

Key words: calibration „in situ“, gas sensors, gas analytical instruments

\begin{abstract}
Motivation and Results
The development of the ecological monitoring and analytical support of industrial technological processes is impossible without the application of high-precision gas analyzers. On the other hand, this high precision, especially in a range of ppm and ppb concentrations, can not be reached without periodical recalibration of gas sensors and analytical instruments. It is very important to perform this recalibration "in situ", just in the place, where the sensors and instruments are installed, that is in laboratories, factories, and ecological monitoring systems. The application of gas cylinder for such calibrations is not convenient, because of high cost and relatively high weight of this cylinder.
\end{abstract}

Moreover, the precision of these calibration gas mixtures prepared in cylinders or volumes made of metals, glass, plastics, etc., is not sufficient, if the concentration of target gas is below $\sim 0.01$ vol. \%. This decrease in the precision is related with the adsorption of gas components on the surface chemical reactions of active gas components (nitrogen oxides, hydrogen sulphide, chlorine, hydrogen chloride and many others) with the material of cylinder walls, and with very big difference in partial pressures of target gas and diluting gas component, leading to multiple gas dilution necessary for the preparation of gas mixture.
Therefore, preparation of this mixture becomes expensive and not precise.

It is also clear that the precision of calibration system should be higher than the precision of instruments to be calibrated. This means, in particular, that not a simple gas cylinder, but a whole gas installation including gas conditioning, humidification, mass flow controllers, supplying gas mixture with a preset flow rate must be used for the calibration of gas sensors and gas analytical instruments.

It is also very convenient, if purified ambient air instead of air in gas cylinder would be used in this installation for the preparation of gas mixtures.

For these calibrations, a dynamical method of gas mixture preparation using diffusion source of target gas was developed (Fig. 1). In this instrument, target gas diffuses through the walls of gas source with a constant rate, and this diffusion gas flow is diluted with carrier air flow. Desirable precision of gas mixture concentration ( $1-4 \%$ rel.) is obtained by the application of mass-flow controllers, use of temperature controlled $\left( \pm 0.1^{\circ} \mathrm{C}\right)$ chamber with diffusion gas source, and by the application of software taking into account gas viscosity as a function of its temperature and exact composition. 
The diffusion source of gases is a sealed Teflon container filled with liquefied target gas (organic and inorganic fluorides and chlorides, $\mathrm{NO}_{2}$, $\mathrm{NH}_{3}$, amines, mercaptanes, hudrocarbons, etc., Fig. 2 and 3). We developed several types of diffusion sources made of PTFE, chlorine containing thermo-softening fluoropolymers, and other fluoropolymers fabricated as gas permeable tubes and ampoules with taps made of the same material, gas-tight ampoules with gas permeable membranes, etc.

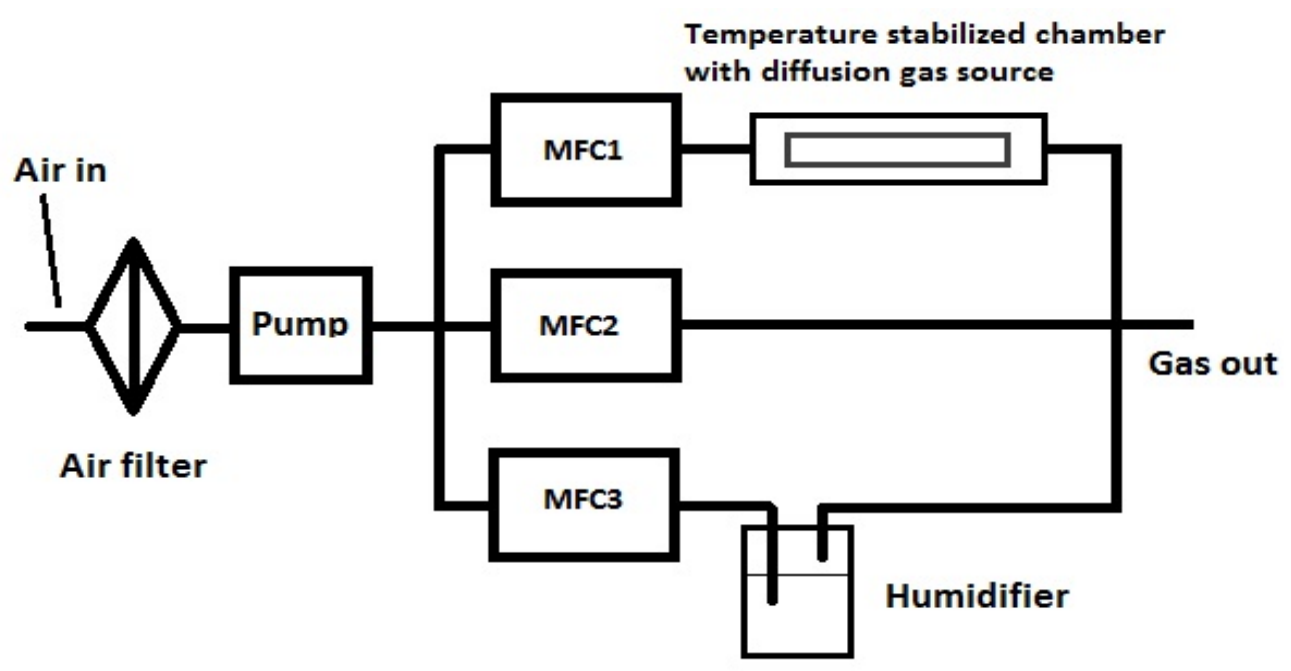

Fig. 1: Scheme of the dynamic set-up with diffusion type gas source for the calibration of gas sensors and analysis instruments. MFC are mass flow controllers.

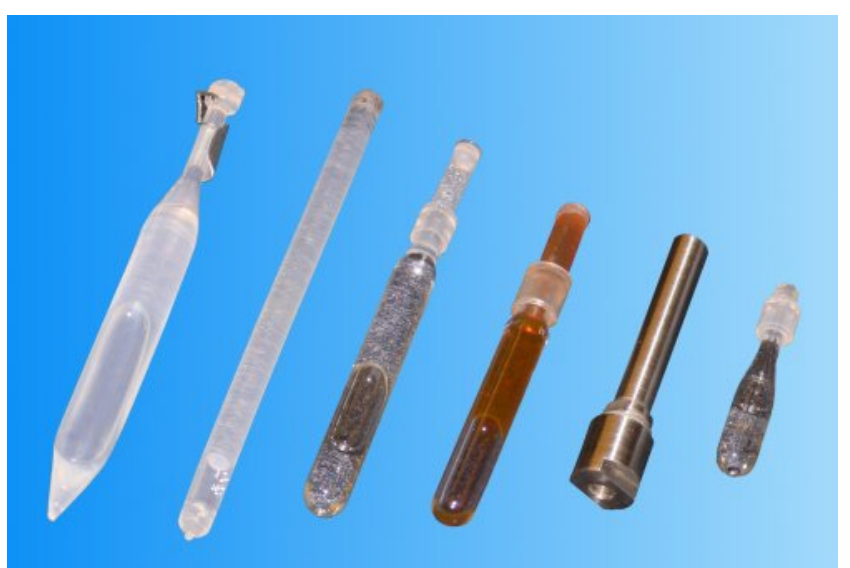

Fig. 2: Diffusion type gas sources (NH3, NO2, HCl, etc.) produced by "PGS-Analytic", Kirovochepetsk, Russia.

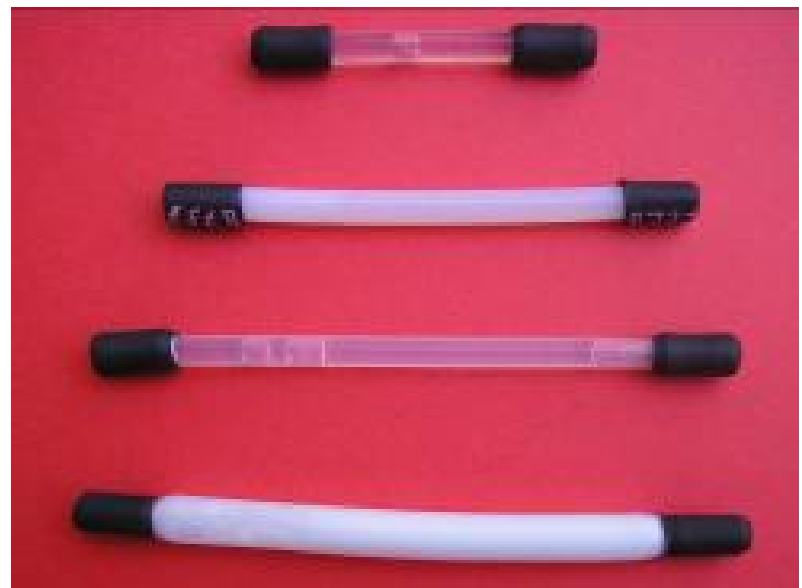

Fig. 3: Diffusion type gas sources produced by the company "Fine permeation" srl, Messina, Italy [1]. 
The quantity of gas $G$, passing through the wall of this container (the wall thickness is $X$ and their area is $S$ ) during time $t$ is determined by Fick's law:

$\mathrm{G}=P \cdot(\Delta p / X) \cdot S \cdot t$,

where $P$ is the diffusion permeability of the wall and $\Delta p$ is the pressure of vapor at temperature $T$. The flux of the diffusing substance is equal to $Q=G / t$. The calibration of the fluoropolymer container remains constant, if there is certain amount of target compound in the container and, therefore, if the pressure of this compound inside the container is constant.

This flux is diluted by carrier gas (dry air) passing through the temperature stabilized cell with diffusion gas source. The quantity of liquefied gas in a diffusion source is sufficient to maintain a certain flux and, therefore, the concentration of target gas for long time during several months or even some years.

The calibration of gas permeable container is performed by the measurement of deficit of mass after several hours of heating of the container in the temperature stabilized chamber of the gas mixing installation or in an external oven stabilizing the same temperature. The permeability of the container can be recalculated to another temperature, because the diffusion rate as a function of temperature is pre-measured for the materials of containers. Therefore, the stabilization of different temperatures of the container enables the variation of diffusion rates in a large range.

The stability of gas diffusion method of the preparation of calibration gas concentrations was studied in detail using hydrogen fluoride, trifluorotrichloroethane (Freon-113), and some other inorganic and organic compounds. Calibration curves that is deficit of mass of the container made of modified Teflon filled with dry target substance as a function of time and diffusion rate as a function of temperature of the container are parallel to each other and linear.

To obtain a desirable concentration of calibrating gas having certain humidity, the setup contains two additional gas lines controlled by mass flow controllers: the line of diluting air and the line of air with $100 \%$ relative humidity. Air passing through these lines dilutes gas flow passing through the cell with diffusion gas source. In this way, it is possible to obtain calibration gas mixture with humidity in a range from 10 to $90 \% \mathrm{RH}$. This possibility is very important for the calibration of gas sensors tested as a rule at humidity of $50-60 \% \mathrm{RH}$.

The calibration set-up can work in both autonomous and computer-controlled regimes. In the last case, computer is used for programming the set-up, for reading, displaying and saving the protocol, containing information about gas concentration as a function of time, errors, it is used also for the diagnostics of failures in the operation of the set-up.

The set-up is powered by accumulator ( 4 hours of autonomy) and uses for gas dilution ambient air purifier by passing trough the filter. The autonomy of the installation is reached, because of the use of special low power consuming mass-flow controllers made in Yoshkar-Ola (Mari-El, Russia). These mass flow controllers can operate at low pressure drop (less than $0.5 \mathrm{~atm}$ ), therefore it is possible to use less power air pump. Another factor is the application of small diffusion chamber with inner diameter equal to the diameter of diffusion gas source and the application of very efficient thermal insulation with a material consisting of evacuated micro-spheres. This material, first, is an efficient thermal isolation and, on the other hand, does not adsorb target gas and, therefore, does not disturb calibration gas concentration.

The design of the calibration system enables its application as a source of low concentrations of water vapor in air with dew point down to $-90^{\circ} \mathrm{C}$. In this case, the diffusion source of water is put to the diffusion channel of the installation, and dried air is used in its inlet as a carrier gas.

In the case, when the power consumption of the installation is the most important factor, the humidification channel can be excluded, and the humidity of diluting air is equal to the humidity of ambient air. This could be also important for some application, when the gas sensor or system must be calibrated just under ambient conditions, but not at a fixed humidity of air.

The portable set-up is equipped an original diffusion gas chamber, which slides out of the set-up body only in the operation state of the set-up. As a result, the size of the installation is of about $400 \times 250 \times 250 \mathrm{~mm}$. The weight is of about $7 \mathrm{~kg}$. It is powered by a accumulator, which could be recharged from car electrical network (12 V).

The typical target gas concentration, at which the application of the dynamic set-up is most suitable, is from $10^{-3}$ to $10^{-1}$ vol. $\%(10-1000$ $\mathrm{ppm})$. In this case, the precision of gas concentration is of $1-4 \%$ rel. Optionally, it is possible to use the installation to set up gas concentrations in a range of $100 \mathrm{ppb}-1000$ 
ppm.

Tab. 1: Characteristics of the portable dynamic set-up with diffusion type gas source.

\begin{tabular}{|l|l|l|l|}
\hline & $\begin{array}{l}\text { Environics } 4000 \\
{[2]}\end{array}$ & "Microgas F" & $\begin{array}{l}\text { Portable } \\
\text { dynamic set-up }\end{array}$ \\
\hline $\begin{array}{l}\text { Average error of gas } \\
\text { concentration }\end{array}$ & $<3$ rel. \% & $<5$ rel. \% & $<5$ rel. \% \\
\hline Flow rate range, $\mathrm{cm}^{3} / \mathrm{min}$ & $50-1000$ & $20-1000$ & $100-600$ \\
\hline Material of gas lines & Viton & Teflon & Teflon \\
\hline Software & Yes & yes & yes \\
\hline Weigt, kg & 10 & 6 & 4 \\
\hline Average power consumption & $260 \mathrm{~W}$ & $200 \mathrm{~W}$ & $80 \mathrm{~W}$ \\
\hline Number of gas channels & 4 & $1-6$ & 3 \\
\hline Gas diffusion channels & No & yes & yes \\
\hline Gas humidification & Yes & yes & yes \\
\hline Carrier gas & $\begin{array}{l}\text { Synthetic air } \\
\text { from cylinders }\end{array}$ & $\begin{array}{l}\text { Synthetic air from } \\
\text { cylinder }\end{array}$ & Atmospheric air \\
\hline Ambient temperature, ${ }^{0} \mathrm{C}$ & $+15 \ldots+35$ & $+15 \ldots+25$ & $-10 \ldots+30$ \\
\hline Autonomy & No & no & yes \\
\hline
\end{tabular}

The characteristics are given in the Tab. 1. In this table, the characteristics of an desk-top, stationary installations "Environics 4000" [2] and "Microgas-F" are compared with the characteristics of the portable installation.

\section{Conclusion}

In this work, it was shown that the dynamic methods of the preparation of calibration gas mixtures in a range of $100 \mathrm{ppb}-1000 \mathrm{ppm}$, especially in low concentration range (below $100 \mathrm{ppm}$ ) are more precise than the static methods. Gas diffusion gas sources can be applied for the preparation of calibration gas mixtures of gases, which could be obtained at room temperature as a liquid.

Portable gas mixing installation developed in this work is a precise and suitable instrument, which can be used for the calibration of gas sensors and gas analytical instruments "in situ". The precision of gas mixture preparation is of about 1 - $4 \%$ rel.

\section{Acknowledgements}

The results of this work were obtained partially in the framework of the joint S3 project cofinanced by the program FP-7 of the European Union (grant agreement \# 247768) and Russian ministry of science and education (State contract \# 11.519.11.3006).

\section{References}

[1] http://www.finepermeation.it

[2] http://www.environics.com 